\title{
Guest editorial: Statistical inference for assessing and monitoring natural resources
}

\author{
Lucio Barabesi $^{1}$ • Lorenzo Fattorini ${ }^{1}$
}

Received: 18 January 2018 / Revised: 25 January 2018 / Published online: 19 February 2018

C Springer Science+Business Media, LLC, part of Springer Nature 2018

This special issue comprises a selection of presentations at the First International Workshop on "Statistical Inference for Assessing and Monitoring Natural Resources", held at the Department of Economics and Statistics of the University of Siena on November 10-11, 2016. The goal of this two-day workshop was to gather environmental statisticians and quantitative naturalists from Italy and abroad, in order to propose and discuss methodological advances as well as to apply previously established methodologies in the field of environmental research. In accordance with the workshops purposes, the selected papers cover the whole field of statistical methods for environmental studies, from modelling and prediction to sampling. The following is a brief description of the content of each contribution.

As to the model-based approach to inference, the first paper of the special issue is authored by Bernard Pelletier and Pierre Dutilleul. The authors propose an update and exhaustive survey of the coregionalization analysis with a drift, reviewing the methodological aspects of the topic, as well as the available software. They also consider various applications of the method, such as the analysis of plant species diversity in a forest stand of Southern Québec (Canada) and the co-evolution of polygonal and scalloped terrains at the surface of Mars. The paper authored by Enrica Caporali, Valentina Chiarello and Alessandra Petrucci deals with extreme hydrological events, analyzing the annual maxima of daily rainfall in the Tuscany region (Central Italy). In adopting a generalized extreme-value model, the authors target the catchment area of the Arno river. A second group of model-based contributions is focused on analyzing

Handling Editor: Pierre Dutilleul.

\footnotetext{
$凶 \quad$ Lucio Barabesi

lucio.barabesi@unisi.it

1 Department of Economics and Statistics, University of Siena, Piazza San Francesco, 7, 53100 Siena, Italy
} 
species diversity. Francesco Dotto and Alessio Farcomeni perform species abundance estimation by introducing a generalized Chao estimator with measurement errors and external information for the enumeration of a whale shark population located in the South Ari atoll (Maldives). Camille Coron, Clément Calenge, Christophe Giraud and Romain Julliard propose a novel statistical procedure to monitor the relative species abundance for different habitat types, using opportunistic data. The technique is illustrated with two datasets of common bird observations in Aquitaine (South-Western French region). Still in the topic of species diversity, Linda Altieri, Daniela Cocchi and Giulia Roli propose a novel descriptive measure of entropy for environmental spatial data. The authors assess it on the dataset of the tree species of a completely-mapped forest in the Barro Colorado Island (Panama).

The remaining papers of this special issue belong to the design-based approach to inference, and deal with sampling strategies for environmental populations. Maria Chiara Pagliarella, Piermaria Corona and Lorenzo Fattorini compare spatiallybalanced sampling with unbalanced stratified sampling, for the purpose of assessing forest change. The two sampling designs are compared on an artificial forest map generated from a real satellite map of Landsat forest/non-forest classification performed in Sardinia island (Italy). Stefano Antonio Gattone, Paolo Giordani, Tonio Di Battista and Francesca Fortuna propose a two-phase sampling strategy that conjugates the adaptive-cluster double sampling with the principle of post-stratification. The proposed strategy is applied to sample an epiphytic lichen community in a forest area of the Northern Apennines (North of Italy). For their part, Federico Andreis and Marco Bonetti present a sampling strategy that joins some features of sequential sampling and adaptive sampling, in such a way that units with desired characteristics are overrepresented. The method is used to analyze the topsoil lead concentration in a flood plain of the Meuse river (The Netherlands). Finally, Maria Michela Dickson, Diego Giuliani, Giuseppe Espa, Marco Bee, Emanuele Taufer and Flavio Santi show how to deal with the presence of positional errors in spatial sampling schemes, when the locations of population units are inaccurate. In this paper, the efficiency loss of the schemes is investigated in a simulation study based on two real populations of longleafpine trees in Southern Georgia (USA) and of turkey-oak trees in an agro-forest hill domain in Lazio (Central Italy). Starting from perfect positioning, the authors simulate two typical examples of coarsening that frequently impact the positioning of tree populations.

We would like to thank warmly all the contributors to this special issue for the enthusiasm with which they participated in the project and for the efforts that they dedicated to the preparation of their manuscript. We are also beholden to the distinguished scholars who acted as reviewers; their careful and thoughtful work is gratefully acknowledged. Finally, our sincere thanks go to Michelangelo Vasta, Head of the Department of Economics and Statistics for supporting the workshop, to Alessio Pollice, Coordinator of the GRASPA-SIS group for giving the patronage and for joining the Scientific Committee, and to Pierre Dutilleul, Editor-in-Chief of Environmental and Ecological Statistics, for accepting to attend the conference with a keynote talk, joining the Scientific Committee and hosting this special issue in EEST. We would also like to thank Alessio and Pierre for their suggestions and support during the organization of the workshop and the entire process of putting together this special issue. The 
final thanks are due to Marzia Marcheselli, Caterina Pisani and Rosa Maria Di Biase for joining the Organizing Committee and managing the practical issues regarding the workshop.

Lucio Barabesi is Full Professor of Statistics at the Department of Economics and Statistics of the University of Siena. His current topics of interest include sampling theory for environmental and social surveys, random variate generation, statistical evaluation of scientific research, as well as statistical fraud detection. He has been Associate Editor of Journal of Agricultural, Biological and Environmental Statistics and CoEditor of Statistical Methods and Applications. At the present, he is Associate Editor of Environmental and Ecological Statistics.

Lorenzo Fattorini is Full Professor of Statistics at the Department of Economics and Statistics of the University of Siena. His field of interest is sampling theory with applications to environmental surveys and ecological diversity analysis. He is Associate Editor of Environmetrics and Environmental and Ecological Statistics, and from 2006 to 2009, he has been Coordinator of the Italian Working Group on Sample Survey Methodology. 